\title{
Inspection of Lettuce Water Stress Based on Multi-sensor Information Fusion Technology
}

\author{
Hongyan Gao, Hanping Mao, and Xiaodong Zhang \\ Key Laboratory of Modern Agricultural Equipment and Technology, \\ Ministry of Education \& Jiangsu Province, Jiangsu University, Zhenjiang 212013, China \\ ghy06@126.com
}

\begin{abstract}
Characteristics of reflection spectrum, multi-spectral images and temperature of lettuce canopy were gained to judge the lettuce's water stress condition which could lead to a precise, rapid \& stable test of lettuce moisture and enlarged the models' universality. By the extraction of lettuce's multi-sensor characteristics in 4 different levels, quantitative analysis model of spectrum including 4 characteristic wavelengths, characteristic model of multispectral image and CWSI were established. These multi-sensor characteristics were fused by using the BP artificial neural network. Based on the fused multisensor characteristics, the lettuce moisture evaluation model was established. The results showed that the correlation coefficient of multi-spectral images model, spectral characteristics model and information fusion model were in turn increased, the correlation coefficients were respectively $0.8042 、 0.8547$ and 0.9337. It was feasible to diagnose lettuce water content by using multi-sensor information fusion of reflectance spectroscopy, multi-spectral images and canopy temperature. The correct rate and robustness of the discriminating model from multi-sensor information fusion were better than those of the model from the single-sensor information.
\end{abstract}

Keywords: Lettuce, Water stress, Information fusion.

\section{Introduction}

Leaf stomata conductance, leaf water potential, transpiration rate, plant stem diameter changes and soil moisture, etc. indirectly illustrated the crop water stress and water requirement. Or the plant water stress is determined using dry weight method by collecting in vivo samples. The traditional testing methods have the shortages of low precision and affecting crop growth and it is not conducive to the promotion application, because in sampling and data analysis are time-consuming. Modern diagnostic methods are mainly spectroscopy, visual images and canopy temperature. However, canopy reflectance spectrum and images have interactions as nitrogen, water and leaf area index, spectrum and image are affected by the crop canopy structure, environmental factors and others. Therefore, a single detection method can not accurately and comprehensively explain the water stress. The discussion group proposes inspection of lettuce water stress based on the multi-sensor information fusion technology. 
Multi-sensor information fusion might comprehensively process multi-source information which comes from some different sensors, so it can obtain more accurate and reliable conclusions [1]. Multi-sensor information fusion can greatly avoid the limitations of a single sensor and improves the performance of the system [2]. Because the sensors provide some uncertain information, multi-sensor information fusion technology is essentially a non-deterministic reasoning and decision-making process [3].

Multi-sensor information fusion can be divided into three different layers which were the decision layer fusion, feature layer fusion and the raw data layer fusion [4]. The research is the optimization and combination of spectra, images, and canopy temperature, because the spectrum analyzer, machine vision systems and other equipments are abstracted into different types of sensors, the features are different and collect different physical quantities, the information pattern and span are comparatively large, environment and objectives with time-varying features, crop characteristics are complex, so it does not suitable for the decision layer fusion and the raw data layer fusion. More practical option is feature layer fusion, the fusion not only retain a sufficient number of original information, but also achieve a level of data compression, contributes to real-time processing[5].The concept of feature layer fusion is different features gather to form the new summary feature set, and then making decisions accordingly[6].

The research obtains lettuce canopy spectrum, image information, canopy temperature and environmental temperature and humidity, etc. Then establish spectra model, mage model and the water stress index model. Ultimately, used BP neural network training samples and verification, water stress conditions on the lettuce is rapidly and non-destructively inspected.

\section{Experimental Design and Sample Training}

\subsection{Instrument and Equipment}

Spectrum measuring equipment is the United States ASD FieldSpec ${ }^{\circledR} 3$ handheld portable spectrum analyzer, the range $350 \sim 2500 \mathrm{~nm}$; at $350 \sim 1000 \mathrm{~nm}$, sampling interval is $1.4 \mathrm{~nm}$, resolution is $3 \mathrm{~nm}$; in $1000 \sim 2500 \mathrm{~nm}$, sampling interval is $2 \mathrm{~nm}$, resolution is $10 \mathrm{~nm}$. High-precision analytical balance weighs the quality of whole lettuce, accuracy is $0.1 \mathrm{mg}$. Canopy multi-spectral image utilizes MS-3100 multi-spectral digital progressive scan camera, imaging spectral range is $400 \sim 1100 \mathrm{~nm}$, resolution is $1039 \times 1392$. Canopy temperature utilizes the TI50 infrared thermal imaging instrument, the range is $-20 \sim 305^{\circ} \mathrm{C}$, accuracy is $0.07^{\circ} \mathrm{C}$.

\subsection{Samples Training}

Experiment started in Jiangsu university modern agricultural equipment and technology Venlo-type greenhouse. The variety is Italian anti-bolting lettuce. According to the Yamazaki Nutrient solution, the samples were divided into four levels, each level had 12 lettuce, so the predict set had 24 samples, calibration set had 20 samples. Four levels were: Group 1(W1) ensure adequate water were supplied throughout the 
growing season; Group 2 (W2), 3 (W3), 4 (W4) irrigated the standard formula of $75 \%$ $50 \%, 25 \%$ concentrate.

\subsection{Experiment Design}

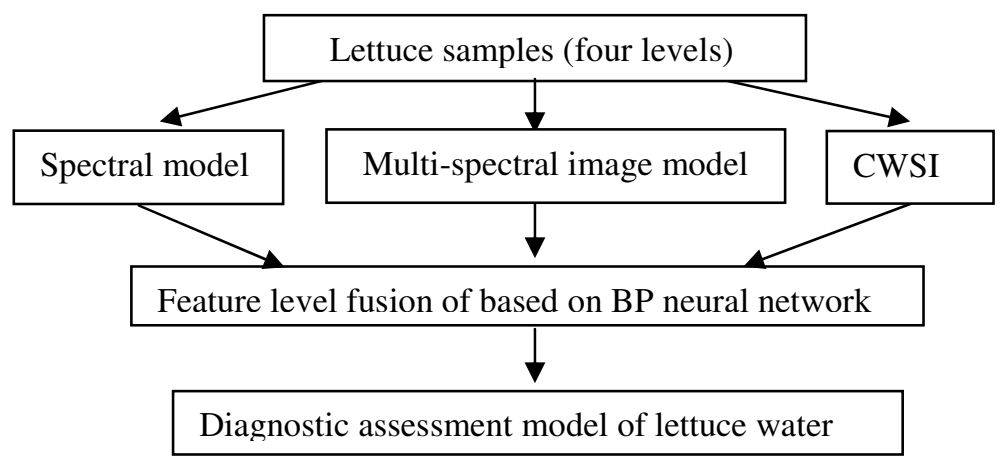

Fig. 1. The flow chart of lettuce water stress inspection based on multi-sensor information fusion technology

\section{Results and Analysis}

\subsection{The Quantitative Analysis of Lettuce Moisture Content Based on Spectrum Technology}

Fig. 2 showed the lettuce canopy reflectance spectra in the different water stress. Combined with previous studies of discussion group, and referenced to the USDA researchers came to the main biochemistry components of the spectral absorption characteristics [7], wavelength sensitive of water-related mostly concentrated in the near infrared band. As Fig. 2 shown, spectral reflectance of lettuce had significant difference in different water stress at the water sensitive bands.

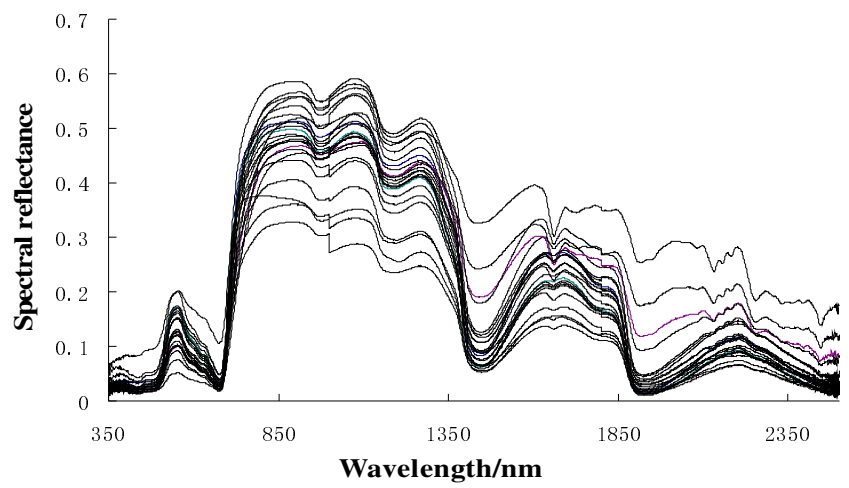

Fig. 2. Lettuce canopy reflectance spectra under different water stress 
In order to eliminate offset and drift which caused the spectral bias, highlight the hidden information and identification of samples. The first order derivative was carried out on the spectrum and conducived to extract characteristic wavelength. In first, all spectral points were divided into 4-sensitive bands: 1220-1300nm ,1410$1490 \mathrm{~nm}, 1620-1700 \mathrm{~nm}, 1900-1970 \mathrm{~nm}$, then removed the wavelength by stepwise regression[8] and got the sensitive wavelength of related to the lettuce canopy water stress: $1267 \mathrm{~nm} 、 1443 \mathrm{~nm} 、 1661 \mathrm{~nm} 、 1921 \mathrm{~nm}$. So as to eliminate the impact of multicollinearity, so the four wavelengths for partial least squares regression analysis[9], When two principal component score were extracted, cumulative contribution rate greater than 0.85 . Ultimately obtained PLS regression model based on four sensitive wavelengths $\left(\mathrm{X}_{\mathrm{i}}\right)$ :

$$
\mathrm{y}=467062-378643 X_{1}-299485 X_{2}-299034 X_{3}+943.02 X_{4}
$$

Then 20 samples of spectral data tested the model, the correlation coefficient between dry water content of lettuce canopy measured and predictive value was 0.8547 .

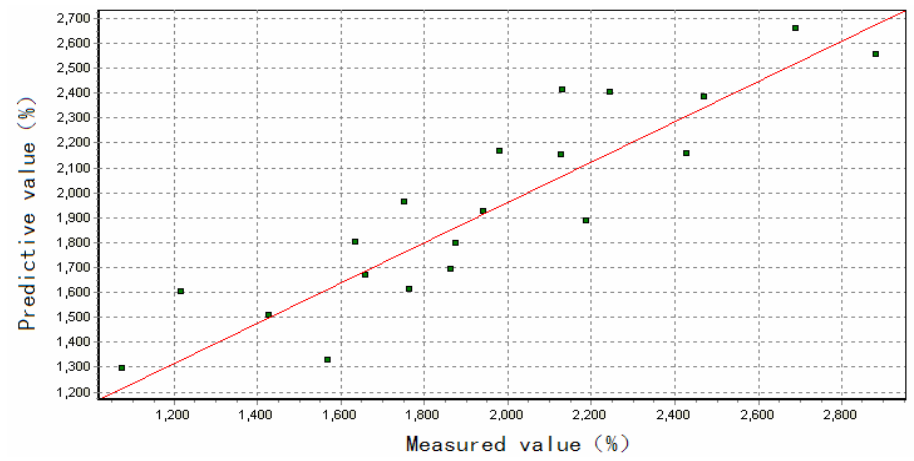

Fig. 3. Lettuce canopy reflectance spectra under different water stress

\subsection{The Quantitative Analysis of Lettuce Moisture Content Based on Multi-spectral Imaging Technology}

The six channels lettuce canopy images were simultaneously acquired by the MS3100 multi-spectral digital progressive scan camera, they were R、G、B、RGB、IR and CIR. This method not only contributed to extract image features of all the independent channels, but also easily achieved multi-spectral image pixel-level operation and integration(Image registration was not required).

Image processing based on MATLAB software. Median filtering method of $3 \times 3$ window would eliminated isolated noise points, reduced the image blurring, so it was used for image preprocessing. And two-dimensional maximum entropy segmentation was used for background segmentation; this method preserved more image information of crop canopy and contributed to the image feature extraction. Experiment used gray feature extraction method, finally $\mathrm{AIR}_{810}$ and $\mathrm{AIR}_{940}\left(\mathrm{AIR}_{\mathrm{k}}\right.$ is near infrared 
spectroscopy $810 \mathrm{~nm}$ and $940 \mathrm{~nm}$ canopy image mean gray value) significantly correlated to lettuce canopy water content. Establishing lettuce canopy water content forecast model by SPSS 13.0 for multiple linear regression analysis, including AIR $_{810}$ and $\mathrm{AIR}_{940}$ image features variable:

$$
y=24.764+124.729 \text { AIR }_{940}+63.775 A^{\prime} R_{810}
$$

Then 20 samples of spectral data tested the model, the correlation coefficient between dry water content of lettuce canopy measured and predictive value was 0.8042 .

\subsection{Canopy Water Stress Index (CWSI) Model Establishment}

The TI50 infrared thermal imaging instrument obtained lettuce canopy temperature and real-timely monitored environmental temperature and humidity. According to the CWSI empirical model by Idso in the literature [10], as follows:

$$
\begin{gathered}
C W S I=\frac{\left(T_{c}-T_{a}\right)-\left(T_{c}-T_{a}\right)_{\Pi}}{\left(T_{c}-T_{a}\right)_{u l}-\left(T_{c}-T_{a}\right)_{\Pi}} \\
\left(T_{c}-T_{a}\right)_{\Pi}=A+B \times V P D \\
V P D=0.611 \times e^{\frac{17.27 \times T_{a}}{T_{a}+237.3}} \times\left(1-\frac{R H}{100}\right) \\
\left(T_{c}-T_{a}\right)_{u l}=A+B \times V P G
\end{gathered}
$$

In the formula: Tc-the crop canopy temperature, ${ }^{\circ} \mathrm{C}$; Ta-air temperature, ${ }^{\circ} \mathrm{C}$; $(\mathrm{Tc}-\mathrm{Ta})_{\Pi}$-lower limit of the difference temperature between canopy and air, ${ }^{\circ} \mathrm{C}$; $(\mathrm{Tc}-\mathrm{Ta})_{\mathrm{ul}}$-limit of the difference temperature between canopy and air, ${ }^{\circ} \mathrm{C}$; VPD-air vapor pressure deficit, hPa. A, B-experience factor; VPG-the difference of air saturated vapor pressure between when the air temperature were Ta and $\mathrm{Ta}+\mathrm{A}, \mathrm{hPa}$.

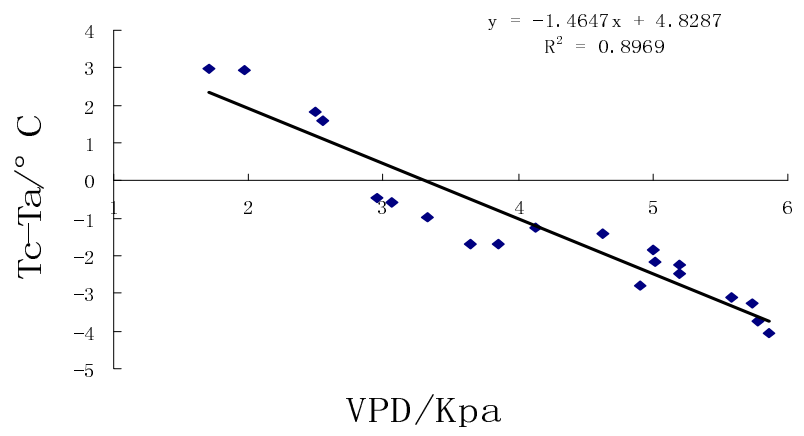

Fig. 4. The relationship of (Tc-Ta) and VDP 
The model of difference temperature between canopy and air by data analysis:

$$
T_{c}-T_{a}=4.8287-1.4647 \mathrm{VPD}
$$

In the condition of full water supply, when VPD was 5.86, the difference temperature between canopy and air was minimum, it regarded as lower limit of CWSI. So $(\mathrm{Tc}-\mathrm{Ta})_{\Pi}$ was -3.7544 . When lettuce was severe water stress, canopy temperature reached the maximum during the experiment, it regarded as upper limit. So $(\mathrm{Tc}-\mathrm{Ta})_{\mathrm{ul}}$ was $1.3241\left({ }^{\circ} \mathrm{C}\right)$.

Lettuce CWSI model:

$$
\text { CWSI }=\frac{|\mathrm{Tc}-\mathrm{Ta}+3.7544|}{5.0785}
$$

\subsection{The Model Based on BP Neural Network Information Fusion}

BP neural network has strong fault tolerance, distributed, storage, self-learning, adaptive, self-organization, nonlinear dynamic capabilities and handle complex environments[11].Therefore, using better self-learning and adaptive capacity, lettuce water stress condition was evaluated by BP neural network.

Table 1. Samples predicted values and the measured values

\begin{tabular}{cccc}
\hline $\begin{array}{c}\text { Sample } \\
\text { Number }\end{array}$ & $\begin{array}{c}\text { Predictive } \\
\text { Value }(\%)\end{array}$ & $\begin{array}{c}\text { Measured } \\
\text { Value }(\%)\end{array}$ & $\begin{array}{c}\text { Relatively } \\
\text { Error }(\%)\end{array}$ \\
\hline 1 & 2984.269 & 2812.366 & 6.112 \\
2 & 2712.595 & 2508.462 & 8.138 \\
3 & 2440.624 & 2604.845 & 6.304 \\
4 & 2402.126 & 2598.000 & 7.539 \\
5 & 2290.337 & 2499.381 & 8.364 \\
6 & 1934.597 & 2161.101 & 10.481 \\
7 & 1852.465 & 2093.596 & 11.518 \\
8 & 1839.795 & 1721.290 & 6.885 \\
9 & 1791.997 & 1623.837 & 10.356 \\
10 & 1780.012 & 1968.000 & 9.552 \\
11 & 1710.706 & 1603.889 & 6.660 \\
12 & 1661.028 & 1798.400 & 7.639 \\
13 & 1690.231 & 1864.688 & 9.356 \\
14 & 1577.302 & 1704.605 & 7.468 \\
15 & 1603.096 & 1428.125 & 12.252 \\
16 & 1687.403 & 1490.943 & 13.177 \\
17 & 1575.115 & 1449.184 & 8.690 \\
18 & 1436.798 & 1625.636 & 11.616 \\
19 & 1206.933 & 1415.647 & 14.743 \\
20 & 1081.009 & 1174.524 & 7.962 \\
Average & & & 9.240 \\
\hline
\end{tabular}


The research used a 3-layer structure of BP neural network for feature level data fusion. There were lettuce canopy reflectance spectra characteristic wavelength $1267 \mathrm{~nm}, 1443 \mathrm{~nm}, 1661 \mathrm{~nm}, 1921 \mathrm{~nm}$ and the image feature parameter AIR $_{810}$, AIR $_{940}$, CWSI as the input, dry basis moisture content measured as the output. Error index and the training step were respectively sited to 0.001 and 0.05 , hidden nodes was 10 . Using BP neural network to predict the same test set (Table 1). The average relative error of predicted and measured values was $9.24 \%$, correlation coefficient $\mathrm{R}$ was 0.9337 .

\section{Conclusion}

Object of study choose four different moisture content of lettuce, lettuce canopy water stress was evaluated by spectral characteristics, image feature information, canopy temperature and Environmental temperature and humidity.

Spectral analysis model and the image model were established, then verifying the model, the correlation coefficient between water content of lettuce canopy measured and predictive value were 0.8547 and 0.8042 .

The results showed that lettuce canopy water stress evaluation method based on the spectrum, multi-spectral image and the CWSI of multi-sensor information fusion technology was feasible, and the correlation coefficient was 0.9337. Model of accuracy and stability were higher than a single information model. Results of the research for multi-sensor information fusion technology could regarded as reference to the rapid and accurate detection of lettuce water.

\section{Acknowledgements}

This work was supported by a grant from the National High Technology Research and Development Program of China (863 Program)(No.2008AA10Z204 and 2008AA102208), "333 Talent Project" in Jiangsu Province.

\section{References}

1. He, Y., Wang, G., Lu, D., Peng, N.: Multi-sensor data fusion and applications. Electronic Industry Press, Beijing (2007)

2. Xiao, W., Li, X., Li, P., Feng, Y., Wang, W., Zhang, J.: Near infrared spectroscopy and machine vision information fusion soil moisture detection. Transactions of the CSAE 25, 14-17 (2009)

3. Ma, G., Zhao, L., Li, P.: Based on Dempster Shafer evidence of multi-sensor information fusion technology and its application. J. Modern Electronic Technology 19, 41-44 (2009)

4. Yan, H., Huang, X., Wang, M.: Multi-sensor data fusion technology and its application. J. Sensor Technology 24, 1-4 (2005)

5. Chen, Q., Zhao, J., Cai, J.: Based on near infrared spectroscopy and machine vision information fusion technology of multi-judge the quality of tea. J. Transactions of the CSAE 24, 5-10 (2008)

6. Wang, R.: Information fusion. Science Press (2007) 
7. Pu, R., Gong, P.: Hyper spectral remote sensing and its application. Higher Education Press, Beijing (2000)

8. Zheng, Y., Zhang, J., Chen, X., Shen, X., Zhang, T.: Based on stepwise regression of nearinfrared spectral information extraction and model. J. Spectroscopy and Spectral Analysis 24, 675-678 (2004)

9. Wang, H.: Partial least squares regression method and its application. National Defense Industry Press, Beijing (1999)

10. Cui, X., Xu, L., Yaun, G., Wang, W., Luo, Y.: Based on the temperature of the summer maize canopy water stress index model study. J. Transactions of the CSAE 21, 22-24 (2005)

11. Xiong, Y., Wen, Z., Wang, M.y.: Based on neural network spectral recognition system design and analysis. J. Spectroscopy and Spectral Analysis 27, 139-142 (2007) 